\title{
Family Planning 2020 and Nepal's Pledge
}

\author{
Archana Amatya, ${ }^{1}$ Ganesh Dangal ${ }^{1}$ \\ 1Journal of Nepal Health Research Council, Kathmandu, Nepal
}

Family planning 2020 is a global partnership which has been started after the 2012 London meeting on Family planning (FP) with the aim of improving the FP services to women and girls in the poorest countries. Achieving the FP2020 goal is critical to ensuring universal access to sexual and reproductive health and rights by 2030 as part of Sustainable Development Goals. The core global partners include Bill and Melinda Gates Foundation, DFID, UNFPA and USAID. In the summit, more than twenty countries committed to revitalize their efforts on family planning. ${ }^{1}$ By 2016 , the partnership expanded which included 40 countries, highlighting a marked increase in political commitment from governments as well as growing interest across diverse sectors. This partnership will help to support women and girl's rights to decide voluntary for themselves, on when and how many children they wish to have. FP 2020 works with the multiple partners including the government, civil society and researchers to make modern methods of FP more available and accessible by 2020.It also addresses the issues of the barriers in terms of society, culture as well as policy, financing and delivery. ${ }^{1,2}$ Among those who made the commitments, one in four countries have developed and endorsed their countries costed implementation plan, another third have increased their allocation of the national budget for FP and half have advocated for FP in some way and held national family planning conferences in their respective countries. ${ }^{1}$

In Nepal, the increase in the use of contraception since 2011 has been contributed by the increased use of traditional methods. Nepal has a stagnated prevalence of modern contraceptives (mCPR) of $43 \%$ and unmet need of $24 \%$, which remains high. ${ }^{3}$ This indicates that Nepal is facing a lot of challenges to meet the Sustainable Development Goal (SDG) target for 2017 of reducing the unmet need for family planning to less than $22 \%$. The existence inequalities in accessing family planning and demand for FP and that the proportion of long-acting and reversible contraceptive acceptance is low remains a huge test for the country to meet the set targets. If all married women who want to space or limit their children were to use family planning methods, the CPR (all methods) would increase to $76 \% .^{3}$ In this context, Nepal will benefit from the FP 2020 commitment by increasing partnership and visibility at the global level for recognition of its work in family planning \{ Costed Implementation Plan (CIP) on family planning: 2015-2020 within the Nepal Health Sector Program III: 2015-2020\}, developing a resource mobilization plan to implement the CIP and monitoring the results.

Moreover, global partnership will present prospects that can help Nepal identify the available and required resources, capacity building needs and other important needs to revitalize family planning programme in the country. Along with this, Nepal will also become a part of global community with experts and advocates on FP. ${ }^{1}$ Additionally Nepal also embraces important strengths in terms of a strong national coordination mechanism (FP sub-committee) and a strong support mechanism for demand generation by several partners. However, there exists multiple barriers and opportunities that either prevent or enable access to FP. Power, governance and accountability, women's participation and leadership are some factors that influence the outcomes and capacity of key players to deliver FP. ${ }^{1}$ Few important challenges that we face are inefficient public procurement system which creates delay and leads to frequent stock outs and inadequate; low quality data in terms of accuracy, and timeliness. The quality of FP counseling services and lack of skilled health workers to provide quality FP have always remained as constraints in Nepal.

In July 2016, the Government of Nepal shared an update on progress in achieving its policy, financial and program and service delivery commitments to FP2020. ${ }^{1}$ Nepal has made pledges as part of FP2020 to address the above barriers, including implementation of CIP 
on family planning (2015-2020) to formulate policies and strategies to work ahead. It has also pledged to address family planning access for adolescents, youth, those living in rural areas, migrants and other marginalized groups and to improve the regulatory framework to promote public-private partnerships.

Implementing FP2020 commitments will make it possible to transform family planning provisions, and extend high quality services to reach the unreached. Making ourselves accountable and working with a range of actors can support and encourage the governments and service providers to do better to meet their commitments, leveraging a scale of impact which would be unachievable by alternative interventions. This will also lead to a tangible change in the global monitoring of Family planning. This aggressive monitoring at the country level will be an important step in helping adjustments at the programmatic level and also show the way for other investors in terms of developing their strategies. The government of Nepal has committed to take the leadership in making sure that women and girls are not barred from making decisions on the number and timing of having children has also focused on providing universal access to quality in FP services. At the national level there is a dire need to multi-sectoral approach to reach our targets and for the implementation of CIP so that no one is left behind.

\section{REFERENCES}

1. Family Planning 2020 [Internet]. [Cited 2017 Aug 11]. Available from: http://www.familyplanning2020.org/

2. WHO Medical eligibility criteria for contraceptive use [Internet]. WHO. [Cited 2017 Aug11].available from:ttp: / / www. who.int/reproductivehealth / publications/family_planning/MEC-5/en/

3. Ministry of Health, Nepal; New ERA; and ICF. Nepal Demographic and Health Survey 2016: Key Indicators. Kathmandu, Nepal: Ministry of Health, 2017. 